\title{
Intersetorialidade e participação social na gestão da educação permanente em saúde em um município de pequeno porte do Rio Grande do Sul: percepções de um Núcleo Municipal de Educação em Saúde Coletiva
}

\section{Intersectoriality and social participation in the continuing education in health management in a small municipality of Rio Grande do Sul: perceptions of a Municipal Health Education Nucleus}

\author{
Paola Lucca Pizutti ${ }^{1}{ }^{1} \bowtie$, Alexandre do Nascimento Almeida ${ }^{2}{ }^{2}$, Luzia Fernandes Millão ${ }^{3}{ }^{3}$ \\ ${ }^{1}$ Secretaria de Estado da Saúde do Rio Grande do Sul, Centro Estadual de Vigilância em Saúde, Porto Alegre, RS, Brasil. \\ ${ }^{2}$ Universidade Federal de Ciências da Saúde de Porto Alegre, Departamento de Educação e Humanidades, Porto Alegre, RS, Brasil. \\ Universidade Federal de Ciências da Saúde de Porto Alegre, Departamento de Enfermagem, Porto Alegre, RS, Brasil.
}

RESUMO

Objetivo: Este artigo objetivou descrever percepções relacionadas à intersetorialidade e à participação social na gestão da política de Educação Permanente em Saúde (EPS), observados por profissionais da saúde participantes do Núcleo Municipal de Educação em Saúde Coletiva (NUMESC) de um município de pequeno porte no Rio Grande do Sul.

Materiais e Métodos: Por meio de abordagem qualitativa, com metodologia de pesquisa-ação, foram realizadas oito reuniões com o NUMESC, que foram gravadas, transcritas e submetidas à análise de conteúdo temática. Para organização do material produzido foi utilizado o software Atlas.ti.

Resultados: Este artigo descreve duas categorias temáticas encontradas: "Movimentos para o trabalho em rede intersetorial e o intercâmbio de experiências" e "Entraves para a efetiva participação social".

Conclusão: Na primeira categoria, os profissionais afirmaram que foram feitos movimentos para um trabalho envolvendo outros setores do município, o que demandou rupturas em modos de fazer, porém trouxe resultados positivos na gestão da EPS. Na segunda categoria, observaram que, embora prevista nas normas que regem o Sistema Único de Saúde (SUS), a participação social ainda é pouco expressiva nos espaços de gestão municipal e praticamente inexistente na gestão da educação em saúde.

Palavras-chave: educação permanente; estratégia saúde da família; saúde pública; participação social.

Objective: This article aimed to describe perceptions related to intersectoriality and social participation in the continuing education in health (EPS) management observed by health professionals members of a Municipal Health Education Nucleus (NUMESC) in a small municipality in the state of Rio Grande do Sul.

Materials and Methods: Using a qualitative approach, eight meetings were held with the NUMESC. Meetings were recorded, transcribed and submitted to the thematic content analysis. The software Atlas.ti was used to organize the material produced.

Results: This article describes two thematic categories found: "Movements for intersectoral work and the exchange of experiences" and "Obstacles to effective social participation".

Conclusion: In the first category, professionals affirmed that movements were made in order to obtain the involvement of other sectors of the municipality, which required ruptures in traditional ways of doing, but brought positive results in the management of EPS. In the second category, they observed that, although foreseen in the norms that govern the public health system (SUS), social participation is still not very expressive in the spaces of municipal management and practically nonexistent in the management of health education.

Keywords: continuing education; family health strategy; public health; social participation.

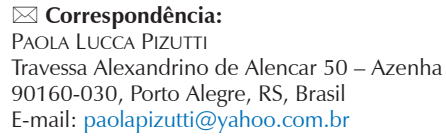




\section{INTRODUÇÃO}

A política de Educação Permanente em Saúde (EPS) no âmbito do Sistema Único de Saúde (SUS) tem o objetivo de promover transformações nas práticas não só de atenção, mas também de gestão e participação social, considerando a complexidade dos serviços de saúde e a necessidade de promover uma postura crítica, reflexiva e propositiva dos trabalhadores da saúde ${ }^{1}$.

Como transformação necessária ao processo de trabalho no SUS, pode-se colocar o desafio da superação do modelo biomédico, ainda predominante nas práticas de saúde, que centraliza o cuidado nas especialidades médicas de modo fragmentado. Para o fortalecimento das ações de saúde, com foco na promoção e prevenção, é necessária a construção gradativa de um modelo pautado na lógica da integralidade na atenção e na clínica ampliada. Nesse escopo, a EPS está presente como uma estratégia de aprendizagem no trabalho que se dá a partir dos problemas cotidianos dos serviços de saúde, que demanda conexões em rede, integralidade do cuidado, gestão efetiva e colegiada² .

A integralidade parte de um projeto ético-político sustentado no reconhecimento de que a vida do outro vale a pena, entra em disputa com o modelo biomédico fortemente arraigado na organização dos serviços de saúde, modelo esse cartesiano, com concepção biológica e mecanicista, especializado na doença, fragmentador do indivíduo, com predomínio de abordagens terapêuticas prescritivas e autoritárias e baixa resolutividade do ponto de vista da saúde integral, pode-se dizer "fiz a minha parte". Como contraponto a esse modelo, está a construção de um novo paradigma de saúde, pautado no plano terapêutico para sujeitos e coletivos biopsicossociais, com saberes e desejos, experiências e na construção de vínculos entre usuários e comunidades com as equipes de saúde, pode-se dizer "vamos fazer juntos" 3 .

De acordo com Sarreta ${ }^{4}$ a EPS se fortalece na procura por parcerias e experiências próximas que possam ser intercambiadas. A complexidade dos problemas na atenção à saúde demanda envolvimento de conhecimentos provenientes de diversos campos e disciplinas do saber, sendo a interdisciplinaridade e a intersetorialidade fundamentais para a construção de caminhos adequados.

A parceria que se almeja para a educação permanente dos profissionais da saúde é de unir setores envolvidos no fazer saúde, articulando essas instâncias de modo a garantir a autonomia de cada um. A realização dessa articulação é desafiadora, pois ao colocar diferentes atores em contato, são evidenciados interesses divergentes e que disputam espaços ${ }^{5}$.
A Lei Federal no 8.142/1990 regulamenta a participação da comunidade na gestão e define que o SUS deve contar com Conselho de Saúde com composição paritária de membros em todas as esferas de governo: união, estados e municípios ${ }^{6}$. A representação popular por meio dos conselhos de saúde vem sendo garantida como espaço para fiscalização de ações do setor saúde pela comunidade, sendo um importante instrumento da população para promover a formulação de políticas para a promoção da saúde como um direito, contribuindo ativamente na definição de prioridades. Entretanto, devido à existência de diferentes concepções e perspectivas metodológicas e políticas, a formação e a articulação dos conselhos de saúde têm sido um desafio em nível nacional, estadual, municipal e local'7.

Um dos pontos críticos da política pública de saúde é a visão bastante disseminada da saúde como objeto de consumo. Elevar a consciência para a noção de saúde enquanto direito requer fortalecimento do controle social e da cidadania. Os conselhos de saúde representam importantes instâncias políticas para promover esta discussão e estimular a ação dos cidadãos no processo de tomada de decisão, sendo que a agenda da EPS para o controle social, em especial a Educação Popular, promotora do trabalho pedagógico problematizador, pode contribuir para a construção de conselhos mais democráticos, participativos e com maior capacidade de articulação e negociação7.

Quando se fala em articulação intersetorial, em linha estreita com a EPS está a noção de quadrilátero da saúde, que traz a importância da interlocução entre gestão, atenção, ensino e controle social para transformação dos serviços de saúde. Nesse escopo, a formação dos trabalhadores articulada ao quadrilátero da saúde é capaz de proporcionar ambiente fértil para a $\mathrm{EPS}^{8}$. É importante considerar que o quadrilátero continua sendo o vértice da EPS, todavia é preciso perceber as multiplicidades que operam nesses setores. Ao mencionar as instituições de ensino, ou de usuários, por exemplo, existem inúmeros universos possíveis, assim como as esferas de gestão política do SUS incluem multiplicidades que vão da gestão setorial, gestão de si, do seu processo de trabalho até a gestão do Ministério da Saúde. Tal como a esfinge descrita por Merhy (2015), qualquer campo da composição básica do quadrilátero pode ser pensado como uma multidão9.

Para a evolução do modelo de saúde acontecer, é preciso questionar permanentemente os modos estabelecidos de produção de saúde, em especial superar as práticas protocolares de núcleos profissionais frente às demandas e necessidades de saúde das pessoas e suas comunidades ${ }^{3}$.

A saúde não pode ser vista como um objetivo estático, e sim processual. O objetivo se constrói gradativamente em um processo de desenvolvimento que pode oscilar durante 
a vida, entre a perda, o equilíbrio ou o ganho de saúde. Os limites e possibilidades de saúde de cada indivíduo são descobertos por meio da construção de uma consciência crítica que acontece em níveis de aprendizagem sobre a realidade social e sobre si mesmo, ao mesmo tempo que demanda responsabilidade do Estado em garantir a base para a qualidade de vida da população. O empoderamento de gestores, trabalhadores e usuários é gerado a partir da consciência crítica, especialmente quando o agir pedagógico em saúde tem a intenção de contribuir para a construção de sujeitos críticos ${ }^{10}$.

Este artigo objetivou descrever percepções relacionadas à intersetorialidade e à participação social na gestão da política de EPS, observados por profissionais da saúde participantes do Núcleo Municipal de Educação em Saúde Coletiva (NUMESC) vinculados à Secretaria Municipal de Saúde (SMS) de Catuípe, município de pequeno porte no Rio Grande do Sul.

Considerando a intersetorialidade e a participação social como estratégicas para a gestão das políticas do SUS, em especial as políticas voltadas à EPS, este artigo descreve a análise de duas categorias temáticas: "Movimentos para o trabalho em rede intersetorial e o intercâmbio de experiências" e "Entraves para a efetiva participação social", temas estes originários da investigação intitulada "Educação Permanente em Saúde como estratégia para o fortalecimento da atenção básica no município de Catuípe: análise e perspectivas".

\section{MATERIAIS E MÉTODOS}

A pesquisa apresenta caráter qualitativo, com base no conceito de pesquisa-ação, que compreende as etapas de planejamento, ação, monitoramento e descrição e avaliação ${ }^{11}$.

O estudo se deu junto ao NUMESC, grupo organizado com a finalidade de realizar a gestão e planejar ações de EPS para profissionais da saúde e usuários do SUS no âmbito da SMS. Para geração dos dados foram realizadas oito reuniões Com o grupo NUMESC sobre a temática da gestão da EPS no município, as quais foram gravadas e as falas transcritas integralmente. O material foi organizado e transcrito com apoio do software Atlas.ti. A partir do material obtido foi realizada análise temática ${ }^{12}$.

A pesquisa ocorreu no período de julho a dezembro de 2015 na Secretaria Municipal da Saúde (SMS) do município de Catuípe/RS, localizado na região Noroeste do Estado do Rio Grande do Sul, com população de 9.477 habitantes. A estrutura da SMS dispõe de duas Unidades Básicas de Saúde (UBS) e quatro equipes de Estratégia de Saúde da Família $(\mathrm{ESF})^{13}$.
O número de participantes das reuniões do grupo NUMESC foi 11 (onze) pessoas, envolvendo profissionais ligados também aos setores de atenção básica (ESF), Núcleo Ampliado de Saúde da Família e Atenção Básica (NASF-ab) e vigilância sanitária no município estudado, entre estes uma enfermeira, uma fiscal sanitária de nível médio, uma cirurgiã-dentista, uma fisioterapeuta, uma educadora física, uma assistente social, duas psicólogas, dois médicos e a pesquisadora.

$\mathrm{Na}$ análise temática de conteúdo, foram encontrados temas que versavam sobre a intersetorialidade e sobre a participação popular da gestão da EPS. No texto final foram destacadas falas que representam os núcleos de sentido encontrados e as falas foram identificadas por códigos $\mathrm{N}$ (de NUMESC) seguidos de números de 1 (um) a 11 (onze) para a proteção da identidade dos participantes.

O projeto de pesquisa foi aprovado pelo Comitê de Ética em Pesquisa da Universidade Federal de Ciências da Saúde de Porto Alegre (CEP/UFCSPA) com CAAE número 45476015.2.0000.5345.

\section{RESULTADOS E DISCUSSÃO}

As categorias temáticas analisadas refletem os "Movimentos para o trabalho em rede intersetorial e o intercâmbio de experiências" e os "Entraves para a efetiva participação social".

\section{Movimentos para o trabalho em rede intersetorial e o intercâmbio de experiências}

As narrativas a respeito das primeiras iniciativas de articulação intersetorial demonstraram que o NUMESC observou melhorias e qualificação no trabalho nesse processo, porém também houve relatos de frustrações em decorrência de não obter receitas prontas e da necessidade de rupturas nos próprios modos de fazer e na própria compreensão sobre EPS.

É possível observar que inicialmente o NUMESC buscava respostas prontas sobre como realizar as ações de EPS, como fazer o plano de ações em EPS no município e quais atividades priorizar. O desenvolvimento do trabalho do grupo trazia cada vez mais questões para reflexão e provocou um sentimento de geração de dúvidas.

\footnotetext{
“Nos sentimos pequenos, que não sabíamos nada, achamos que íamos encontrar as respostas e saímos com mais dúvidas. Sobre como trabalhar a educação permanente, como fazer os intercâmbios, como fazer o plano de educação permanente." (N6)
}

A proposta da EPS é de que as prioridades educativas devem emergir a partir dos problemas da realidade local, 
do cotidiano dos serviços de saúde ${ }^{14}$. Desconstrói-se muitas vezes para reconstruir novos modos de fazer. Houve narrativas que demonstraram o crescente entendimento entre o grupo sobre a construção do processo de trabalho com base na realidade local.

"Não tem receita pronta cada município deve fazer [o plano de educação permanente] conforme a sua realidade, mas foi muito bom trocarmos experiências com pessoas de outros municípios que nos deram ideias. Perguntaram se nós já estudamos textos de educação permanente [risos]." (N7)

O motivo dos risos na fala citada ocorreu como descontração, pois a pesquisadora incentivou a realização de leituras de textos de apoio em EPS durante as reuniões do NUMESC e houve certa resistência do grupo. Entretanto, percebeu-se que com o desenvolvimento do trabalho do NUMESC, mais leituras se faziam necessárias como suporte às práticas.

Diferentes ofertas podem ser trazidas pelo apoiador externo, como a experiência, a lei, o referencial teórico. Para trabalhar como apoiador com o gestor ou com uma equipe da atenção básica é preciso conhecer as diretrizes políticas e institucionais, sendo que as contribuições externas são apresentadas como oferta do apoiador e quem recebe a oferta pode utilizar como julgar adequado, pode incorporar ou não, pode trabalhar junto e discutir em uma perspectiva de composição com a experiência interna ${ }^{15}$.

Destacaram-se narrações sobre a existência de muitos casos de violência subnotificados no município e a respeito da necessidade da articulação com outros setores para um trabalho mais efetivo, com destaque à comunicação inexistente ou difícil entre diferentes setores no município.

"Os agentes comunitários de saúde atendem a um caso de violência doméstica, por exemplo, e se sentem desamparados para agir." (N7)

"Não temos apoio, o agente comunitário diagnostica a violência e muitas vezes nem leva para a equipe por medo. O agressor vai saber que a denúncia veio depois daquela visita." (N3)

De acordo com Nascimento e Deslandes ${ }^{16}$, o enfrentamento à violência é considerado uma questão social e um problema público que demanda ações articuladas entre os setores da educação, saúde, segurança, assistência social e judiciário, como uma agenda pública e intersetorial, uma vez que um setor isolado não consegue enfrentar o problema com eficiência.
O agir solitário enfraquece as possibilidades de enfrentamento ao problema, uma vez que o trabalho, especialmente na atenção básica, deve ser orientado no sentido da equipe e da abordagem multidisciplinar dos casos e da mesma forma, o apoio de outros entes poderia contribuir com ações frente a muitos problemas de saúde ${ }^{8}$.

O compartilhamento dos desafios entre os profissionais da saúde coloca o interlocutor em certa posição de legitimidade para construir as soluções em conjunto, em uma perspectiva de interação pedagógica que além de contribuir com o outro para a mudança de sua prática igualmente o convoca a ser agente de transformações. O aprendizado que decorre da atuação dos profissionais é a potência da EPS como estratégia, uma vez que o encontro e a negociação entre os pares promove o surgimento de novas lideranças no cotidiano do trabalho, bem como de novas formas de fazer que vão sendo construídas e pactuadas, reforçando os elos da rede ${ }^{1}$.

Nesse sentido, destacaram-se as narrativas de rodas de conversa promovidas pelo NUMESC envolvendo as equipes de ESF e outros convidados, como profissionais da educação, assistência social, segurança, judiciário e controle social, denominadas Encontros de EPS, que foram eventos descritos como verdadeiros potenciais de transformação do trabalho. Um destes encontros abordou a temática da violência e como forma de articulação intersetorial em rede foram convidados representantes de entidades ligadas à assistência social e judiciário, além dos profissionais da equipe de saúde municipal.

\footnotetext{
"Temos que pensar de que forma podemos estar mudando a forma de trabalhar e sair do modelo no qual a violência é um problema da segurança pública, do judiciário, de assistentes sociais e psicólogas. É um problema de todos juntos." (N5)
}

A aproximação intersetorial para determinada finalidade potencializa as possibilidades de um desfecho positivo das ações conjuntas. Quando um objetivo é comum, funciona como um dispositivo que é capaz de ativar a rede intersetorial. É necessária a existência de uma intenção comum aos entes que compõem a rede, com força suficiente para que seja estabelecido um elo coletivo com direcionamento a determinados valores ou iniciativas ${ }^{17}$. No caso estudado, houve bastante ênfase em narrativas que evidenciam dificuldades no enfrentamento à violência contra a mulher no município, o que provocou ações no sentido de estreitar vínculos institucionais nos Encontros de EPS propostos pelo NUMESC.

Foram verificados sentidos de busca por articulação intersetorial no trabalho, extraídos das falas dos participantes, 
entretanto, não foram encontrados indícios que mostrassem a operacionalização da lógica da rede hierárquica e organizada. De acordo com Mendes ${ }^{18}$ para se caracterizar a operacionalização na lógica de rede, deve haver uma coordenação, bem como fluxos e pactuações estabelecidos. Evidenciou-se a necessidade de se construir maiores conhecimentos a respeito da articulação intersetorial para que ações como essa não se esvaziem em si e possam de fato disparar processos de rede.

Relatos destacaram que a presença de duas enfermeiras, uma servidora pública vinculada à atenção básica de outro município e outra coordenadora da atenção básica na esfera estadual, em reunião com a equipe de saúde de Catuípe, trouxe novos conhecimentos além de ressignificação de conceitos já trabalhados em equipe:

"Tu viu, já fizemos um intercâmbio! É bom quando vêm profissionais de fora! Quando a gente fala é de casa, não tem o mesmo valor." (N5)

A respeito da temática do intercâmbio de experiências como instrumento de qualificação das ações na Atenção Básica, houve ocorrências de narrativas denotando a importância do intercâmbio, em uma perspectiva do enriquecimento pela troca de conhecimentos. Ao confessar "Quando a gente fala é de casa, não tem o mesmo valor", sob confirmação de todo o grupo, destaca-se a necessidade de trazer eventuais apoiadores externos para colaborar em discussões truncadas ou já esgotadas pela equipe, mas ao mesmo tempo demonstra a necessidade de reafirmar que ainda assim a "prata da casa" continua tendo valor ${ }^{19}$.

O intercâmbio de experiências é exitoso quando permite que se desenvolvam novas ações, novas práticas ou novos significados para as práticas em decorrência da troca realizada e na lógica do intercâmbio, é possível ser apoiador e também ser apoiado por outros profissionais e equipes ${ }^{3}$. O NUMESC vivenciou esses lugares e isso emergiu das narrativas sobre troca de experiências. Destaca-se uma fala que representa a compreensão do intercâmbio e do apoio mútuo em rede:

"Acho que o intercâmbio é uma via de mão dupla, uma hora tu recebe apoio, em outra vira apoiador." (N8)

A fala em evidência demonstra o entendimento sobre a proposta da EPS que se caracteriza por valorizar o conhecimento de todos os atores da rede, em que todos podem contribuir e não necessitam somente ser apoiados, podem ver-se na perspectiva de apoiadores também².

O apoio é uma metodologia com referencial na cogestão que procura contribuir com a gestão participativa e capilarizada e desconstrói a mudança imposta, pois vem para construir a mudança. Na relação do apoio não há saber que se sobrepõe, há uma horizontalidade e se consideram as experiências existencial e institucional. Há uma parte histórica, política, institucional que virá de fora, mas é preciso a experiência, o conhecimento dos usuários, dos trabalhadores, os contexto sejam valorizados em um conjunto como forma de fazer a práxis, de gerenciar as transformações ${ }^{15,20}$.

Com ênfase na lógica horizontal das relações, a fala denota a percepção de uma participante do grupo sobre o próprio trabalho ter se transformado depois de se inserir no NUMESC:

"Antes do grupo existir a gente nem se conhecia, nunca se falava, umas no consultório, outras na unidade, na VISA [Vigilância Sanitária]. Começamos a trabalhar mais juntas, pensando junto. Eu comecei a entender mais do trabalho delas e elas do meu. Eu nem sabia o que elas faziam." (N3)

Esta fala realça o entendimento da importância do espaço de encontro, de diálogo, a conclusão de que houve intercâmbio também internamente no grupo NUMESC, uma vez que os profissionais que participaram das reuniões do grupo eram oriundos de diferentes ESF, do NASF, da VISA e puderam trocar experiências a partir do lugar que cada um ocupa no trabalho.

Nessa ótica, Magalhães ${ }^{17}$ reverencia a potência do encontro para a construção da integralidade da atenção e da gestão do processo de trabalho, enquanto Pinto ${ }^{1}$ observa que embora o discurso seja presente na gestão, na prática é preciso que os processos sejam cada vez mais permeáveis à participação, uma vez que as ações pedagógicas nem sempre conseguem mobilizar mudanças e melhorias no trabalho de forma isolada. Para agenciar as transformações orientadas para melhoria da gestão do trabalho em saúde é necessária articulação política intra e intersetorialmente nos serviços de saúde.

\section{Entraves para a efetiva participação social}

Este tema traz a discussão realizada pelo NUMESC sobre a necessidade percebida pelo grupo em ampliar a participação dos usuários do SUS nas atividades de EPS, bem como na gestão da saúde municipal. É possível perceber a intenção dos entrevistados em envolver mais atores na gestão da EPS.

\footnotetext{
"Tem que achar um meio também. Claro, todo mundo tá aprendendo né, todo mundo tem algo a acrescentar e algo a aprender. Meio de ampliar para a população, não só nós ficarmos com o grupo, mas também de conseguir atingir e envolver a comunidade é importante." (N3)
} 
Verificou-se no grupo NUMESC, a tendência à abertura da gestão do grupo. Pode-se dizer que o grupo era definido, mas a gestão da EPS não era do NUMESC e nem mesmo o grupo era "o dono da EPS", ou seja, aquele lugar estava aberto para outros participantes incluindo a comunidade ${ }^{7}$. A designação dos membros para constituição do NUMESC foi assinada pelo secretário da saúde e também pelo presidente do Conselho Municipal de Saúde (COMUS), representando, dessa forma, uma importante iniciativa entre gestor e controle social no sentido de fortalecer a EPS na área da saúde municipal.

De acordo com a política nacional de EPS, o quadrilátero que envolve gestores, trabalhadores, comunidade de ensino e participação social, deve participar das ações que envolvem EPS ${ }^{8}$. Entretanto, após a designação formal do grupo, narrativas ressaltaram que não houve participação do COMUS nas reuniões do NUMESC, embora os conselheiros tenham sido convidados. A motivação em ampliar o convite aos usuários dos serviços nas unidades de saúde ficou evidente.

"Entre as diversas coisas da organização é importante ligar novamente para os conselheiros do COMUS para convidá-los a participar e ver com a equipe outras pessoas da comunidade, por exemplo, representantes do reassentamento" (N6)

Não obstante a importância da participação popular nas esferas de gestão seja reconhecida, as ações do grupo não conseguiram se traduzir em maior engajamento da comunidade. Nessa análise é preciso considerar que as reuniões do NUMESC e atividades de EPS promovidas na secretaria da saúde ocorreram em horário comercial, entre 08h30min e 17h30min, o que impossibilita a participação de usuários que trabalham nesse horário ${ }^{2,21}$. Foi suscitada a discussão sobre o horário das reuniões, porém não houve adesão à troca de horário, convencionou-se que a reunião de NUMESC é atividade do trabalho e deveria ocorrer em horário de trabalho.

Chamou a atenção nas narrativas, a questão de o COMUS de Catuípe ser um conselho desarticulado e pouco atuante, com pouca participação social, servindo apenas para aprovar os projetos da gestão, como uma mera instância protocolar, por ser obrigatório manter conselho para receber os recursos da União e Estado.

"Tudo se aprova sem critério no conselho. Se o conselho estivesse constituído por pessoas mais conscientes seria diferente." (N5)

De acordo com Prado, Sarmento e Costa ${ }^{22}$, a iniciativa de se criar espaços de aproximação entre os serviços de saúde e os cidadãos, tem o objetivo de promover o diálogo para uma participação popular mais efetiva, que pode demandar a repactuação de processos e fluxos assistenciais e a implementação de mudanças nos processos de gestão e atenção. Tendo o diálogo como ferramenta estruturante da relação, os conselhos e demais espaços de participação popular tem o potencial de se deslocar do lugar passivo de aprovação de relatórios de gestão, para o delineamento de uma relação de trabalho participativo, com a experiência partilhada de informações e ideias.

O diálogo e a gestão colegiada entre diferentes atores suscitam debates e conflitos, sendo necessário identificar o conflito também como possibilidade de produção de novas realidades e as rodas e espaços de intercâmbios de informação e experiências como instrumento de gestão e educação ${ }^{23}$. Reflexões a respeito da caminhada do grupo NUMESC e seu impacto na equipe, bem como a necessidade de participação da comunidade:

"Acho que é uma caminhada muito legal que vai avançando, conseguimos organizar a casa em uma postura mais horizontal, as pessoas estão se sentindo parte, tem lugar para colocar opinião, tem mais voz e daqui a pouco começa a incluir a comunidade controle social, conselho. E aí começa a abrir." (N10)

Os Conselhos de Saúde são órgãos colegiados e permanentes de gestão com caráter deliberativo, que devem ter em sua composição membros representantes do governo, prestadores de serviço, profissionais de saúde e usuários. Atuam na priorização de estratégias e exercem o controle e fiscalização inclusive econômico e financeiro da execução da política de saúde no nível correspondente ${ }^{6}$. Na fala fica evidenciada a dificuldade de conseguir maior participação popular nas instâncias:

"Em todos os seminários e reuniões que participamos o pessoal tem cobrado a presença do controle social. Temos que ver isso, como poderia se inserir mais no NUMESC, mais junto com a gente... Como fortalecer o COMUS que praticamente não existe participação dos usuários." (N5)

De acordo com $\mathrm{Cruz}^{7}$, os conselhos locais diferem dos anteriormente citados, por serem mais próximos da população, o que favorece a participação da comunidade e igualmente se torna um desafio, pois com esta relação de proximidade, os problemas do cotidiano ficam evidenciados. Desenvolver habilidades para dialogar em equipe incluindo os usuários é essencial, uma vez que é necessário negociar e gerir conflitos oriundos dessas relações.

Os conselhos são importantes para a concretização de uma concepção ampliada de saúde, da promoção do 
diálogo e integralidade e para isto é imprescindível haver incentivo por parte do gestor público às iniciativas locais de controle social e educação permanente dos conselheiros. O fortalecimento do SUS também passa pelo fortalecimento da participação social nas instâncias formais de gestão, assim como no cotidiano das unidades de saúde através do vínculo entre usuário e unidade, um desafio que parece demandar esforço contínuo de gestores e equipes de saúde.

\section{CONSIDERAÇÕES FINAIS}

Com a realização deste estudo, foi possível verificar as percepções de participantes de um núcleo voltado para a gestão da EPS, sobre articulações intersetoriais, intercâmbios de experiências e a participação da comunidade nos espaços de gestão. É possível concluir que as atividades apresentaram potencial de mudança nas práticas de saúde, especialmente nos momentos em que se realizaram intercâmbios e ações intersetoriais, favoreceu-se a aprendizagem coletiva e o estabelecimento de vínculos e parcerias que colaboram para qualificação das ações na atenção à saúde. No entanto, verificou-se que existem obstáculos que dificultam uma maior participação popular, sendo eles, desde a conscientização da comunidade sobre a importância de participar até questões de incompatibilidade de horários. É importante que iniciativas como as do NUMESC, que buscam aproximação com a comunidade, tenham continuidade para que as práticas de saúde se aproximem cada vez mais das demandas da comunidade em que se inserem.

\section{REFERÊNCIAS}

1. Pinto HA. Política nacional de educação permanente em saúde: aprender para transformar. In: Gomes LB, Barbosa MG, Ferla AA (org.). A educação permanente em saúde e as redes colaborativas: conexões para a produção de saberes e práticas. Atenção Básica e Educação na Saúde. Porto Alegre: Rede Unida; 2016. 23-65.

2. Castro CP, Campos GWS. Apoio Institucional Paideia como estratégia para educação permanente em saúde. Trab. Educ. Saúde. 2014;12(1):29-50. https://doi.org/10.1590/S198177462014000100003

3. Seixas CT, Merhy EE, Baduy RS, Junior HS. La integralidad desde la perspectiva del cuidado en salud: una experiencia del Sistema Único de Salud en Brasil. Salud Colect. 2016;12(1):113-23. https://doi.org/10.18294/sc.2016.874

4. Sarreta FO. Educação permanente em saúde para os trabalhadores do SUS. São Paulo: Cultura Acadêmica; 2009. https://doi.org/ 10.7476/9788579830099

5. Santos DAF, Mourão L, Naiff LAM. Representações Sociais acerca do Trabalho em Equipe. Psicol. cienc. prof. 2014;34(3):643-659. https://doi.org/10.1590/1982-3703000802013
6. Brasil. Presidência da República. Lei no 8.142, de 28 de dezembro de 1990. Dispõe sobre a participação da comunidade na gestão do Sistema Único de Saúde (SUS) e sobre as transferências intergovernamentais de recursos financeiros na área da saúde e dá outras providências. Brasília: Diário Oficial da União 31dez 1990; Seção 1:4-5.

7. Cruz PJSC, Vieira SCR, Massa NM, Araújo TAM, Vasconcelos ACCP. Desafios para a participação popular em saúde: reflexões a partir da educação popular na construção de conselho local de saúde em comunidades de João Pessoa, PB. Saude soc. 2012;21(4):1087-1100. https://doi.org/10.1590/S010412902012000400025

8. Ceccim RB, Feuerwerker LCM. O quadrilátero da formação para a área da saúde: ensino, gestão, atenção e controle social. Physis. 2004;14(1):41-65. https://doi.org/10.1590/S010373312004000100004

9. Merhy EE. Multidão: esfinge da saúde pública, lugar de inflexão, ideias do bem comum. Saude soc. 2015;24(1):44-54. https://doi. org/10.1590/S0104-12902015S01004

10. Lopes R, Tocantins FR. Promoção da saúde e a educação crítica. Interface: Comunic., Saúde, Educ. 2012;16(40):235-46. https:// doi.org/10.1590/S1414-32832012005000009

11. Tripp D. Educação e Pesquisa. 13 ed. São Paulo: Ed. São Paulo; 2005.

12. Minayo MCS. O desafio do conhecimento: pesquisa qualitativa em saúde. 14 ed. São Paulo: Hucitec; 2014.

13. Catuípe. Prefeitura Municipal de Catuípe: A cidade [Internet]. 2015 [capturado 2015 Ago 29]. Disponível em: http://www. catuipe. rs.gov.br/a-cidade

14. Ceccim RB. Educação permanente em saúde: desafio ambicioso e necessário. Interface: Comunic., Saúde, Educ. 2004;9(16):161-168. https://doi.org/10.1590/S1414-3283 2005000100013

15. Righi LB. Apoio matricial e institucional em Saúde: entrevista com Gastão Wagner de Sousa Campos. Interface: Comunic., Saúde, Educ. 2014;18(Supl 1):1145-50. https://doi.org/10.1590/180757622014.1146

16. Nascimento AF, Deslandes SF. A construção da agenda pública brasileira de enfrentamento da violência sexual infanto-juvenil. Physis. 2016; 26(4):1171-1191. https://doi.org/10.1590/s010373312016000400006

17. Magalhães HMJ. Redes de Atenção à Saúde: rumo à integralidade. Divulg. saúde debate. 2014;52:15-37. Disponível em: http:// cebes.org.br/site/wp-content/uploads/2014/12/Divulgacao52.pdf

18. Mendes EV. Comentários sobre as Redes de Atenção à Saúde no SUS. Divulg. saúde debate. 2014;52:38-49. Disponível em: http://cebes.org.br/site/wp-content/uploads/2014/12/ Divulgacao-52.pdf 
19. Gomes LB, Merhy EE. Colaborações ao debate sobre a revisão da política nacional de educação permanente em saúde In: Gomes LB, Barbosa MG, Ferla AA (org.). A educação permanente em saúde e as redes colaborativas: conexões para a produção de saberes e práticas. Atenção Básica e Educação na Saúde. Porto Alegre: Rede Unida; 2016.67-92.

20. Campos GWS. Saúde Paideia. 3. ed. São Paulo: Hucitec; 2011.

21. Cecilio LCO. O "trabalhador moral" na saúde: reflexões sobre um conceito. Interface (Botucatu). 2007;11(22):345-351. https://doi. org/10.1590/S1414-32832007000200012

22. Prado EV, Sarmento DS, Costa LJA. O diálogo como estratégia de promoção de participação popular no SUS. Rev. APS.

2015;18(4):424-429. Disponível em: https://aps.ufjf.emnuvens. com.br/aps/article/view/2702/905

23. Cardoso IM. "Rodas de educação permanente" na atenção básica de saúde: analisando contribuições. Saude soc. 2012;21(Supl.1):18-28. https://doi.org/10.1590/S010412902012000500002

\section{Autores:}

PAOLA LUCCA PIZUTTI

Farmacêutica. Mestre em Ensino na Saúde. Especialista em Vigilância Sanitária.

Especialista em saúde na Secretaria de Estado da Saúde do Rio Grande do Sul, Centro Estadual de Vigilância em Saúde, Porto Alegre, RS, Brasil.

Orcid: https://orcid.org/0000-0001-9060-063X

E-mail: paolapizutti@yahoo.com.br

AleXANDRE do NASCIMENTO ALmeIDA

Graduado em Letras. Doutor e Mestre em Letras. Especialista em Gênero e

Sexualidade e em Língua Inglesa. Docente na Universidade Federal de Ciências da

Saúde de Porto Alegre, Departamento de Educação e Humanidades, Porto Alegre, RS, Brasil.

Orcid: https://orcid.org/0000-0003-4485-9944

E-mail: almeida.n.alexandre@gmail.com

LUZIA FERNANDES MILLÃO

Enfermeira. Doutora em Psicologia. Docente na Universidade Federal de Ciências da Saúde de Porto Alegre, Departamento de Enfermagem, Porto Alegre, RS, Brasil.

Orcid: https://orcid.org/0000-0003-3399-7428

E-mail: luziam.ufcspa@gmail.com 